\title{
Retos en la prevención del embarazo adolescente subsiguiente, un estudio desde la perspectiva de madres adolescentes
}

\section{challenges in preventing a subsequent pregnancy in adolescents: perspectives of adolescents' mothers}

\author{
Paola González Nava ${ }^{a}$ \\ (iD) https://orcid.org/0000-0003-4144-8736 \\ E-mail: pao_navaळmsn.com \\ Yesica Rangel Flores ${ }^{a}$ \\ (iD) https://orcid.org/0000-0001-5673-6891 \\ E-mail: yrangelmaestriaळhotmail.com

\section{Eduardo Hernández Ibarra ${ }^{a}$} \\ (D) https://orcid.org/0000-0002-3889-7533 \\ E-mail: eduardo_ibarraळhotmail.com \\ aUniversidad Autónoma de San Luis Potosí. Facultad de \\ Enfermería y Nutrición. San Luis Potosí, San Luis Potosí, México.
}

\section{Resumen}

El presente artículo expone el resultado de una evaluación cualitativa sobre las acciones que los servicios públicos de salud desarrollan para la prevención de embarazos subsiguientes en adolescentes, en un estado en el centro-norte de México. El objetivo fue documentar los obstáculos percibidos para prevenir embarazos subsiguientes en madres adolescentes. La información se obtuvo entre 2016-2018, mediante entrevistas individuales en los domicilios de jóvenes usuarias de servicios públicos de salud. El análisis de la información se hizo a partir de la propuesta de Strauss y Corbin para teoría fundamentada. Las experiencias compartidas por las jóvenes madres fueron analizadas y clasificadas en dos categorías, obstáculos asociados a: (1) competencias profesionales, y (2) a imaginarios morales. Se concluye que las limitaciones más importantes tienen que ver con el hecho de que la estrategia de servicios amigables para adolescentes deja de implementarse en aquellas que han sido madres, sin considerar el impacto biológico y psicosocial que tienen los embarazos subsiguientes en la adolescencia y la necesidad de postergar la reproducción hasta la edad adulta.

Palabras clave: Embarazo en la Adolescencia; Prevención Terciaria; Adolescente; Investigación Cualitativa.

\section{Correspondencia}


This article presents the result of a qualitative evaluation of the actions that the public health services develop for the prevention of subsequent adolescent pregnancies in a state in northcentral Mexico. The objective was to document the perceived obstacles to preventing subsequent teenage pregnancies in teenage mothers. The information was obtained in the period 2016-2018, by individual interviews in the homes of young users of public health services. The analysis of the information was based on Strauss and Corbin's proposal for the grounded theory. The experiences shared by the young mothers were analyzed and classified into two categories, obstacles associated with: (1) professional competences, and (2) with moral imagery. It is concluded that the most important limitations are related to the fact that the strategy of adolescent friendly services is no longer implemented in those who have been mothers, without considering the biological and psychosocial impact that subsequent pregnancies have in adolescence and the need to delay reproduction until adulthood.

Keywords: Pregnancy in Adolescence; Tertiary Prevention; Adolescent; Qualitative Research.
En México como en la mayor parte de América Latina y el Caribe, el embarazo adolescente (EA) representa uno de los desafíos más importantes en materia de salud pública, dado sus impactos biológicos y psicosociales (UNFPA, 2016). En el ámbito biológico por su estrecha vinculación con la mortalidad materna y prematurez extrema (Silva et al., 2018), en lo psicosocial por su íntima relación con la deserción escolar, la inserción de jóvenes madres al empleo precarizado y en general con la contribución de lo anterior a la feminización de la pobreza (Medina; Ortiz, 2018).

Las políticas dirigidas a contener el EA exigen incidir en el problema a partir de los tres niveles de prevención, mediante la prevención primaria, para evitar la experiencia de un primer embarazo; a través de la prevención secundaria, para reducir los riesgos biopsicosociales que conlleva el EA en esta población específica; y finalmente pero no menos importante, la prevención terciaria, con el fin de prevenir embarazos subsiguientes y disminuir lo más posible los riesgos biopsicosociales asociados a la maternidad temprana (Rodríguez, 2012).

Respecto a la relevancia de incorporar la prevención terciaria en el abordaje del EA, cabe recordar que fue en el Consenso de Montevideo sobre Población y Desarrollo donde se señaló la importancia de impulsar estrategias intersectoriales para prevenir el embarazo subsiguiente en la adolescencia, así como garantizar que niñas y adolescente accedan a "la atención prenatal, del parto y posparto, el acceso a métodos anticonceptivos, a las acciones de protección y asistencia y a la justicia” (CEPAL, 2013, p. 9). Pese a este exhorto, la mayor parte del esfuerzo de los Estados en todo el mundo se han centrado en la prevención primaria, con la inclusión de estrategias educativas en sexualidad integral y potenciando las competencias de los recursos humanos en salud para intervenir en la consejería desde enfoques denominados "amigables" (WHO, 2012, p. 56).

Poca menos atención se ha puesto sin embargo, en la prevención secundaria y terciaria, particularmente en la prevención de embarazos subsiguientes, entendiendo estos como aquellos nuevos embarazos, que tienen lugar uno o dos años después de un primer 
aborto o parto en una adolescente (Escobar, 2006) y que implican incluso una mayor amenaza para el desarrollo y bienestar social de las jóvenes. Se ha documentado que, en el ámbito biológico, el segundo hijo de una madre adolescente enfrenta dos veces mayor riesgo de presentar retraso en el crecimiento intrauterino y hasta tres veces más probabilidad de desarrollar muerte neonatal (Mendoza; Arias; Mendoza, 2012). En el ámbito psicosocial, el embarazo subsiguiente se correlaciona positivamente con uniones matrimoniales tempranas que potencian la vulnerabilidad de las mujeres a situaciones de violencia conyugal (Baldwin; Edelman, 2013; Rosas et al., 2017).

La llegada de un segundo o tercer hijo en la adolescencia suele sobrepasar los recursos de las redes de apoyo para asegurar la permanencia de las jóvenes madres en los espacios educativos, lo que deriva en deserción escolar (Villalobos et al., 2015). Las adolescentes con periodos intergénesicos menores de 24 meses enfrentan, por otra parte, mayor probabilidad de malos resultados en el parto y postparto que cuando fueron madres la primera vez (Gonzáles, 2016) y tienen más del doble de posibilidad de rezago educativo que las que tuvieron un solo embarazo (Binstock; Cerrutti, 2005).

En el contexto antes señalado y aunque la razón por la que los Estados enfatizan más en acciones de prevención primaria puede obedecer a que estas implican menor inversión presupuestal, cabe la posibilidad de que la ausencia de acciones dirigidas a la prevención terciaria tuviesen fundamento en representaciones morales, valores y normas sociales que basadas en el estigma históricamente construido sobre las madres adolescentes, desalienten la continuidad de consejería y acompañamiento "amigable" en este grupo (Núñez; Ayala, 2012), no debe demeritarse el hecho de que las políticas en México y en general en América Latina se deciden dentro de sociedades conservadoras (Benavides; Delclós; Consol, 2018).

Esta investigación documenta las experiencias que un grupo de madres adolescentes ha tenido respecto a la prevención de embarazados subsiguientes en el contexto mexicano, donde desde 2015 se implementa la Estrategia Nacional para la Prevención del Embarazo Adolescente (ENAPEA), política a través de la cual el Estado mexicano busca, para 2030, reducir en un $50 \%$ los embarazos en adolescentes de entre 15 a 19 años, y erradicar el embarazo en niñas menores de 15 años (MÉXICO, 2015).

La ENAPEA está conformada por 18 líneas de acción, de las cuales, sólo una se dirige a la prevención terciaria del embarazo adolescente. Las tres actividades que contempla esta línea se desglosan en la Tabla 1.

\section{Tabla I - Actividades dirigidas a la prevención terciaria del embarazo adolescente (prevención de embarazos subsiguientes)}

I. Fortalecer la calidad de la consejería y la oferta de métodos anticonceptivos post-evento obstétrico para adolescentes en

primer y segundo nivel de atención.

2. Reforzar la consejería en planificación familiar post parto a madres adolescentes cuando asistan a citas de control de niño sano o niña sana en el primer nivel de atención.

3. Incorporar a las guías de práctica clínica de la atención del embarazo y puerperio la consejería sobre anticoncepción durante la atención prenatal para adolescentes, con énfasis en las ventajas del espaciamiento de los embarazos y privilegiando la libre elección con la oferta de la gama completa de métodos, incluyendo los ARAP.

Con marco en lo anterior, el objetivo de esta investigación fue evaluar de manera cualitativa, los obstáculos percibidos por un grupo de madres adolescentes, sobre las acciones que los servicios públicos de salud implementan para prevenir embarazos adolescentes subsiguientes.

\section{Metodología}

Evaluación cualitativa enmarcada en la perspectiva de juventudes. La evaluación cualitativa posibilita recolectar información pero en este proceso detonar además procesos reflexivos y 
emancipatorios en los que las personas/ciudadanas se asumen parte de los procesos sociales y políticas gubernamentales y públicas que enmarcan su cotidianeidad, esto posibilita generar condiciones que aportan a la justicia social (Peñaranda, 2015). Por su parte, la perspectiva de juventudes permite explicar de qué forma las relaciones de poder limitan la autonomía de las personas jóvenes en el ejercicio de sus derechos, - en este caso particularmente de los sexuales -, a partir de un elemento estructural como la edad (Ramiro; Alemán, 2016).

Durante el periodo 2016-2018 se trabajó con madres adolescentes de dos de los municipios con mayor tasa de embarazo adolescente en un estado en el centro-norte de México. Las informantes fueron 12 jóvenes contactadas a través del PROMAJOVEN, un programa de apoyo social que otorga becas escolares para adolescentes embarazadas o jóvenes madres, con el fin de evitar la deserción escolar. La razón por la que se trabajó con jóvenes adscritas a este programa es porque este concentra una cantidad considerable de madres adolescentes, además de que convocarlas a través del programa les generó confianza para participar en la investigación.

La información se recolectó a través de entrevistas individuales, observación no participante y llenado de diarios de campo, lo que posibilitó la triangulación de datos (Angrosino, 2012, p. 61). La observación participante y no participante se realizó en dos de los foros que semestralmente oferta el PROMAJOVEN, al primero se acudió de manera anónima con la finalidad de documentar lo que se abordaba en estos encuentros, así como las dinámicas que las jóvenes vivían en estos eventos; al segundo se asistió en calidad de investigadoras, se informó a las jóvenes sobre el estudio que se realizaría y se convocó su participación, procurando que la información del proyecto fuera concisa y evitando brindar información que posibilitara a las potenciales informantes hacer prejuicios sobre lo que se pretendía encontrar.

Las jóvenes fueron visitadas en sus domicilios para obtener los consentimientos informados y apegar el estudio a los estándares éticos y legales nacionales e internacionales en materia de investigación en salud. El estudio se consideró de riesgo mínimo, - conforme lo establece la Ley General de Salud de México -, y contó con la aprobación y monitoreo del Comité de Ética de la Facultad de Enfermería y Nutrición de la Universidad Autonóma de San Luis Potosí (registro CEIFE-2017-223).

Se llenó un diario de campo tanto en los foros como en las entrevistas, la finalidad fue registrar las incidencias metodológicas y teóricas que surgían en cada encuentro y que sirvieron en la etapa de análisis para interpretar y construir los datos. La entrevista individual se realizó con base a un guion semiestructurado que se construyó a partir de las acciones que establece la ENAPEA para el sector salud, en lo que respecta específicamente a la prevención terciaria.

En función del criterio de saturación teórica, se determinó pertinente suspender la recolección de información tras la entrevista número doce, una vez que la información se evidenció redundante y las relaciones entre las categorías de análisis mediante comparación constante quedaron bien establecidas para garantizar la lógica interna de los datos. Las entrevistas se audiograbaron y fueron transcritas en totalidad con apoyo del programa Otranscribe. Las transcripciones fueron analizadas, codificadas y categorizadas desde la propuesta de Strauss y Corbin (Strauss; Corbin, 2002). Se realizó la triangulación de las categorías identificadas, consensuando la lógica de las categorías y sus relaciones entre todos los integrantes del equipo de investigación. Las matrices de relación entre las categorías se sustentaron en argumentos teórico conceptuales propios de la prevención terciaria.

\section{Resultados}

\section{Caracterización de la población informante}

La media de edad fue 17 años. El 83.3\% vivía en unión libre y el $16.4 \%$ estaban separadas de los padres de sus hijos. El 41.6\% había concluido la educación básica, el 50\% estudiaba la secundaria y el 8.3\% cursaba un nivel técnico-medio superior. En cuanto a sus antecedentes reproductivos, $9(83.3 \%)$ eran madres de un soolo hijo, 2 (16.6\%) tenían dos hijos y $1(8.3 \%)$ cursaba su segundo embarazo. Las jóvenes que tenían más de un hijo así como la que estaba embarazada habían suspendido sus estudios. 
En las mujeres que tenían más de un hijo o estaban actualmente embarazadas, el periodo intergenésico se situó en una media de 14 meses. En el momento de las entrevistas, el 91.6\% refirió contar con algún método de Planificación Familiar (MPF), de estas, 9 (75\%) usaban el implante subdérmico, 1 (8.3\%) DIU de Cobre y 1 (8.3\%) DIU hormonal, 1 no usaba método porque cursaba el embarazo.

Las experiencias compartidas por las jóvenes madres fueron analizadas y clasificadas en tres categorías, obstáculos asociados a: (1) competencias profesionales, (2) políticas institucionales e (3) imaginarios morales.

\section{Obstáculos asociados a competencias profesionales}

Se identificó que la totalidad de las participantes no tenían claridad sobre los riesgos que implica un embarazo adolescente subsiguiente. Pese a que dijeron haber recibido la indicación de no volver a embarazarse, al profundizar sobre la consciencia desarrollada en torno al riesgo que esto implica, ninguna pudo describir los eventos indeseables (biológicos o psicosociales) asociados a un segundo embarazo en la adolescencia.

En la consulta me dijeron [personal médico y de enfermería] que era muy riesgoso, me explicaron las edades del embarazo o algo así, que debía haberme embarazado a partir creo que de los veintiuno [años], me dijeron que estaba en riesgo, ahíme le pusieron en rojo, "embarazo en riesgo" algo así "alto riesgo", entonces me dijeron que era muy riesgoso que me volviera embarazar así tan seguido... exactamente no sé que pueda pasar, pero sí me dijeron que era riesgo. (Entrevistada no.6)

La postergación de un nuevo embarazo figura más como una prescripción que como una decisión informada que les compete ejercer. Narrativas como la anterior fueron comunes y reflejan no soolo una falta de consciencia del riesgo sino la ausencia de competencia del personal sanitario para comunicar el riesgo obstétrico en esta población en particular.

Se identificó por otra parte, que si bien la mayor parte de las informantes hacía uso de un método de acción prolongada en el momento de las entrevistas, la mayoría dijo estar no segura, convencida o conforme con el método implementado. Además, más de la mitad refirió haber aceptado la colocación de un método que no había elegido y la mayoría se dijo no complacida del método usado.

Otro de los hallazgos relevantes tiene que ver con el hecho de que el método anticonceptivo que estas jóvenes deciden inicialmente, está fuermente influenciado por las experiencias que escuchan de otras mujeres cercanas a su red social, dentro de una realidad en la que no se aborda la consejería dentro de las consultas prenatales. Todas las informantes narraron haber "aceptado" la colocación de un método anticonceptivo en la sala de parto o durante su estancia en puerperio, cursando condiciones fisiológicas y emocionales que no les permitían comprender con claridad la ventaja de un método sobre otro.

En el tenor de lo antes señalado, la actitud percibida respecto al desempeño del personal de salud en la sala de parto o de puerperio fue más narrada como un acto de coacción y rutina que de consejería. Así lo muestra la siguiente narrativa:

Pues nada más pasaban [personal de enfermería] y me decían que con que me iba a cuidar, a cada rato pasaban, y a todas les decía que con el implante del brazo, y volvían a pasar y me preguntaban lo mismo, y otra vez, otra vez, pero no me decían nada más de ese implante, hasta que ya pasaron y me dijeron "Ya te vamos a poner el implante", y nada más, no me mencionaron nada, de cómo cuidarlo o así, nada más así. (Entrevistada no. 8)

\section{Obstáculos asociados a políticas institucionales}

La meta con las mujeres adolescentes que han sido madres, no debería limitarse a la colocación de un método reversible de acción prolongada (ARAP) y que aunque por supuesto que es un avance enorme, la actuación debía centrarse en ofrecer una consejería que considere los imaginarios para protegerse frente a la posibilidad de un embarazo subsiguiente.

La consejería basada en un modelo intercultural "amigable" debe incorporar a las madres adolescentes y aplicarse como parte de su seguimiento en el puerperio tardío y en las consultas de vigilancia al 
crecimiento y desarrollo de sus hijos. En el sentido anterior y sin embargo, se identificó que prevalece una ausencia de seguimiento y consejería posterior a la colocación del método, y que el monitoreo del DIU o implante subdérmico en esta población se realiza exactamente igual y con la misma regularidad que se hace en una mujer adulta, la cual está por demás mencionar, no enfrenta el mismo riesgo reproductivo que una adolescente.

La siguiente narrativa evidencia como las jóvenes son instruidas a regresar a los servicios de salud hasta que les toque "el cambio", a partir de programas que no reconocen la importancia de la consejería continua en esta población específica.

Sí, me dijeron [personal médico y de enfermería] que fuera cada tres años, me dieron el pase para que me lo quitaran y me pusieran otro [Implante subdérmicol por eso no he ido a revisarme... nada más me lo pusieron y me dijeron que a los tres años, se cambiaba y me dieron el pase y ya. (Entrevistada no. 3)

Los servicios "amigables" para adolescentes figuran, según las narrativas de estas informantes, aplicados solo en jóvenes que no han sido madres, basadas en imaginarios que carentes de sustento intercultural, perspectiva de género y juventudes, impiden reconocer que las jóvenes que han sido madres requieren igual que las que no lo han sido, de acompañamiento y atención fundamentada en la sensibilidad y empatía que caracteriza la "atención amigable".

La ausencia de condiciones particulares derivadas de la política de "atención amigable" -desalienta el acercamiento de estas mujeres a los servicios de salud, así como puede verse en la siguiente narrativa:

[¿Vas a revisarte el implante al centro de salud?] Sí, pues me dijeron [personal médico y de enfermería] que cada año, me toca ahora hasta diciembre... Tengo que ir, a las seis de la mañana, a sacar ficha para que me puedan atender, aquí en el centro de salud, es que dicen que supuestamente después ya dejan de atender, que porque ya se acaban las fichas. (Entrevistada no. 7)
Las jóvenes no cuentan con acompañamiento para expresar dudas o insatisfacciones con el método, lo que deriva en su renuncia prematura al método, o bien, en resignarse a vivir la experiencia de su uso en un marco de incertidumbre e incomodidad, lo que a la larga les lleva a vivir la anticoncepción más como sacrificio que como derecho.

Las adolescentes enfrentan otras situaciones que dificultan su acceso a los servicios, muchas tienen que decidir entre asistir a la escuela o buscar el consejo profesional ante las reacciones secundarias de los métodos, esto se torna particularmente grave en aquellas que además cuentan con redes de apoyo insuficientes. Otra limitación tiene que ver con el hecho de que en México los servicios de "Consejería amigable" no cuentan con infraestructura de ludoteca o espacios en los que puedan encargar a sus hijos para acceder a la consulta, dado que este modelo de atención amigable no contempla la demanda de atención por parte de adolescentes madres.

Ya no quise ir, pues es que yo sola con mis hijas, si se me hace así mucho porque me ponían las citas a las tres juntas, porque pues tenía a ella chiquita y a ella un poquito más chiquita y pues no podía, agarraba a una cuando checaban a la otra y pues cuando me checaran a mí quien me las iba a agarrar, por eso. (Entrevistada no. 6)

Aunado a lo anterior, es preciso destacar las dificultades que enfrenta el personal de salud para establecer una relación terapéutica ya no "amigable" sino asertiva y respetuosa con las jóvenes. Varias refirieron haber renunciado a las consultas de seguimientos de sus hijos porque se sentían maltratadas por el personal de los servicios públicos de salud.

Al niño lo llevo a un particular [médico], porque había una doctora que me empezaba a criticar, que no le pongas eso, no le pongas aquello, asíme empezaba a regañar, yo digo que hay formas de decir las cosas, entonces me hubiera dicho, no mira esto y esto, así y así, pero no, lo que hacía era regañarme y gritarme. (Entrevistada no. 9) 


\section{Obstáculos asociados a imaginarios morales}

Otras de las narrativas que predominaron, son aquellas que evidencian la persistencia de imaginarios de tipo moral que directa o indirectamente sostienen formas de discriminación percibidas por las jóvenes madres. La actuación del personal sanitario frente a una adolescente embarazada se enmarca en una postura paternalista que reprende o exige explicación sobre las razones de sus embarazos y que termina por instituirse en una barrera para la comunicación profesional-usuaria.

Hubo unas veces que me dijeron [médicos], "¿y por quéno se cuidó? usted está muy chiquita, al momento de que se alivie le van a decir del riesgo y eso", y yo le decía "Ay pues que quiere que haga, antes no me platicaban de eso y mire lo que está pasando ahorita, ya tengo el niño ¿qué quiere que haga?, ya no puedo regresar el tiempo". (Entrevistada no. 10)

Las jóvenes señalan que la actuación del personal puede llegar a ser violenta, en la búsqueda de obligarlas a protegerse frente a la posibilidad de nuevos embarazos.

Pues se me quedaban viendo porque traía la niña y en eso un muchacho de ahí [no sabe si enfermero, médico o promotor] se me acercó y me dijo "Ten para que te cuides", me dio los preservativos, yo me sentí rara porque la gente... toda la gente me volteó a ver. (Entrevistada no. 8)

Además, el personal de salud suele acudir con los padres y no con ellas para la autorización de un método anticonceptivo de acción prolongada.

En ese momento me dijeron Ipersonal médico, de enfermería y trabajo sociall que me lo iban a poner [el DIU], que era más obligatorio que me lo pusiera porque era menor de edad y entonces ahí la que decidía era mi mamá, y sí, mi mamá fue la que autorizó que método se me pusiera, pero pues yo también en todo momento accedí a que se me pusiera, no me estaba negando. (Entrevistada no. 7)

\section{Discusión}

El propósito de esta investigación fue evaluar los obstáculos percibidos por un grupo de madres adolescentes, en relación a las acciones que los servicios públicos de salud implementan para la prevención de embarazos subsiguientes. Lo primero que resulta necesario mencionar es que esta investigación recupera la perspectiva de un grupo de informantes cuyas características socioculturales, pobreza, baja escolaridad y formalización de vida en pareja -, se han documentado como potenciadoras de vulnerabilidad para embarazos subsiguientes (Jiménes; Granados; Rosales, 2017), pero que aún se encuentran inmersas en el sistema escolar, ya sea en su modalidad escolarizada o no escolarizada, lo cual se ha reconocido como un factor protector frente al embarazo adolescente inicial y subsiguiente (Aléman; Insfrán; Castillo, 2018).

Profundizando sobre las características sociodemográficas de las informantes, resultó particularmente interesante dar cuenta de una íntima vinculación entre el ser madre de más de un hijo o cursar la segunda gestación y la renuncia al proyecto educativo, lo que respalda la hipótesis planteada por otros autores, respecto a que tener más de un hijo en la adolescencia incrementa la posibilidad de no completar los estudios (Barros, 2019). En contraparte, el que la mayor parte de ellas se encontrara viviendo en pareja, desafía las afirmaciones que se han elaborado respecto a que las uniones tempranas desalientan la continuidad del proyecto educativo en jóvenes madres (UNFPA, 2016), en tanto respalda el argumento que otros autores han elaborado respecto a que, cuando existen recursos, la pareja está presente y no existen conflictos familiares derivados de la situación de embarazo, la maternidad temprana no representa un riesgo para la continuidad en el desarrollo social y escolar (Pacheco, 2016).

Por otra parte, si bien la cantidad de participantes es insuficiente para hablar de tendencias estadísticas sobre aceptación y uso de métodos anticonceptivos reversibles de acción prolongada (ARAP) posterior a un evento obstétrico en mujeres adolescentes, es pertinente reconocer que el hecho de que más del 9o\% reportara su uso, 
se coloca muy por encima de lo referido en la última Encuesta Nacional de Salud y Nutrición en México (MÉXICO, 2012), la cual reportó su uso en apenas un $48 \%$, o de los referidos en la Encuesta Nacional de la Dinámica Demográfica (CONAPO, 2016) que reportó su utilización en apenas el 35.2\% en jóvenes con nacimientos subsecuentes y el $30.9 \%$ en las que tienen antecedente de un solo nacimiento.

En el contexto anterior y sin embargo, resulta necesario plantear la hipótesis de que este alto porcentaje de utilización tenga relación con el hecho de que una cuarta parte de las informantes al momento de la entrevista eran madres de más de un hijo o estaban cursando un segundo embarazo, lo que pudo motivar la consciencia (en ellas y/o el personal de salud), sobre la urgencia de dotarles de este tipo de métodos.

Los resultados obtenidos en esta investigación coinciden con lo señalado por Zamberlin et al. (2017), respecto a que las debilidades implícitas en el trabajo en materia de anticoncepción con adolescentes, incluyen aspectos relacionados con la necesidad de fortalecer tanto competencias profesionales como ético-morales en el personal sanitario, así como con el reconocimiento de que existen áreas de oportunidad en políticas de salud e institucionales para hacer real el acceso de las jóvenes a los servicios de salud.

Esta investigación documentó la ausencia de consejería en sexualidad integral para la prevención de embarazos subsiguientes, evidenciando cómo la participación del personal sanitario se limita en "prescribir" un método anticonceptivo y, con esto, incluso hasta un proyecto de vida. Con respecto a este último punto, se convence a las jóvenes madres de aceptar la colocación de algún dispositivo anticonceptivo, señalándoles la impertinencia de tener más hijos por el momento y aunque no se duda de que el fin de realizar el anterior se enmarque en el principio de beneficiencia y de los beneficios que lleva implícito el uso de métodos ARAP, no puede dejar de reconocerse que esta práctica prescriptiva e incluso rutinaria (como es percibido por las propias usuarias) se ejerce más como una política de control natal que como una garantía o tutelaje de su derecho a vivir una sexualidad plena, digna y responsable, como quedó establecido desde la década de los noventa en las Conferencias Internacionales de Viena, el Cairo y Beiging (Valenzuela; Villavicencio, 2015).

La participación que se adjudican las jóvenes respecto a la decisión del método es prácticamente nula, tampoco tienen claridad sobre las razones por las cuales es desaconsejable un segundo embarazo adolescente. Esto último se constituye en un riesgo, pues habiendo estado embarazadas y tenido partos, se han sujetado a dicho riesgo y han resultado invictas del mismo, lo cual deriva en la construcción de una perspectiva positiva de la experiencia (puesto que no murieron) y puede generar lo que en la antropología del riesgo se denomina inmunidad subjetiva, que no es más que una emoción de confianza que les imposibilita comprender la dimensión real del riesgo (Douglas, 1982). El riesgo de un embarazo adolescente puede parecerles menor y desalentar la implementación de estrategias necesarias para evitarlo.

Aunque las adolescentes estén familiarizadas con el término "riesgo" y digan reconocer que un nuevo embarazo sería una "condición de riesgo", de forma consciente no objetivizan las consecuencias que un nuevo embarazo y parto pueden tener, las enfermedades y causas de muerte que se asocian a esto, y menos aún los riesgos sociales que de estos derivan para su presente y futuro.

En esta investigación se documentó como la etapa de monitoreo prenatal se constituye en una "oportunidad perdida" para sensibilizarlas e informarlas sobre el riesgo reproductivo en la adolescencia, así como el papel que la anticoncepción tiene en la reducción de dicho riesgo, situación que ha sido documentada por Zamberlin et al. (2017). En relación a lo antes señalado, resulta necesario puntualizar sobre el papel que se espera que desempeñe el personal de salud en la comunicación del riesgo, no se trata solo de comunicar a las jóvenes la alta probabilidad de malos resultados sino de informarlas en profundidad sobre las estrategias para reducir los riesgos, las cuales incluyen de manera protagónica el uso de métodos de anticoncepción. Solo abordando el riesgo desde estas dos aristas (comunicación del riesgo e información sobre estrategias de protección) es posible potenciar el desarrollo de una agencia ante el riesgo, entendiendo esta desde el marco de Giddens 
(1986, p. 9, traducción propia), como una acción que "implica hacer" en el cuerpo y a través de él, y no solo tener la "intención de hacer".

Hablar de agencia implica deconstruir la actuación que el personal sanitario ha desempeñado históricamente en materia de planificación familiar, así como asumir necesaria la intervención de consejería y dejar de lado la "prescripción” de métodos de planificación familiar. Una participación profesional que reconozca que el acompañamiento que necesitan las jóvenes no puede fundamentarse exclusivamente en lo biológico, sino en el reconocimiento de las condiciones socioculturales que prevalecen en los contextos en que estas jóvenes (y sus parejas) viven su sexualidad, lo que exige como han señalado Gómez et al. (2017) deconstruir el imaginario de que la edad es un condicionante para el uso o no uso de algunos métodos y aceptando que no existe un método anticonceptivo ideal para las adolescentes, aunque reconociendo que los métodos ARAP garantizan una protección más duradera y efectiva, además de que evitan someter a las adolescentes a la gestión de un método de protección en cada encuentro sexual, derivado de esto último la principal debilidad asociada a los métodos ARAP, el hecho de que llevan a las jóvenes a desestimar la necesidad de usar métodos de barrera durante el encuentro sexual y con esto ser más susceptibles de contagiarse de una Infección de Transmisión Sexual (ITS) (Raidoo et al., 2020).

La relación terapéutica que en materia de consejería en planificación familiar es deseable, - desde una perspectiva de las juventudes -, debe ofertarse dentro de un ambiente que brinde información con respeto siempre a la autonomía personal, donde se propicie que la joven cuestione y argumente sus miedos y razones para elegir o no un método, garantizando, en todo momento, que la información que se devuelva a estas usuarias se fundamente en evidencia científica y laica. Solo teniendo lo anterior como preámbulo, será posible para las jóvenes desplegar su agencia sexual y reproductiva.

El personal sanitario deberá considerar en la búsqueda de aconsejar sobre el método más adecuado, no solo la eficacia de este sino las condiciones de que disponen las jóvenes para cumplir con el uso adecuado del mismo, su resiliencia para tolerar o sobrellevar los efectos secundarios y las estrategias con que cuentan para solventar su costo económico, simbólico y social (Sam et al., 2014), todo lo anterior, reconociendo que algunos métodos se representan socialmente como indeseables en relación a la moralidad, integridad y honestidad de las mujeres (Solís; Silva, 2017).

Otra de las áreas de oportunidad que se identificó en esta investigación tiene que ver con el seguimiento que se hace de las adolescentes una vez que han aceptado la colocación de un método anticonceptivo, y que adquiere importancia en virtud de que por su estado de madurez (física y psicosocial) se consideran portadoras de muy alto riesgo reproductivo.

Esta investigación documentó que aun cuando las jóvenes son usuarias en su mayoría de métodos anticonceptivos de acción prolongada (ARAP), aceptan la colocación de estos dispositivos sin garantías de acceso a programas de consejería y acompañamiento profesional en la adaptación (o no adaptación) al método. Las limitaciones que existen para que lo anterior se lleve a cabo son diversas, incluyen cuestiones de tipo administrativas y estructurales. Respecto a las limitaciones administrativas, los horarios de atención suelen ser incompatibles con su permanencia en el sistema escolar, se aprecia además, que el "enfoque amigable" en la consejería se limita exclusivamente a las jóvenes que no han sido madres, situación que también fue documentada en el estudio de Rojas Ramírez et al. (2017). En cuanto a las limitaciones de infraestructura, si bien la literatura señala que los "servicios amigables" garantizan que el encuentro terapéutico transcurra en una atmosfera de intimidad, confidencialidad y libre de interrupciones, sin embargo, se identificó que estos "servicios amigables" no se garantizan para adolescentes que son madres, toda vez que los centros no cuentan con espacios en los que puedan encargar a sus hijos para acceder a los servicios de consulta.

Esta investigación ha documentado, por otra parte, la persistencia de conductas discriminatorias por parte del personal de salud, lo que, de acuerdo a la legislación vigente en México y a tratados internacionales en materia de derechos humanos, 
constituyen acciones violatorias a los derechos sexuales y reproductivos (Taquette, 2013). La narración de encuentros con personal de salud que desde una actuación paternalista juzga, recrimina y exige explicación sobre la vida sexual de las jóvenes, constituye una de las barreras más fuertes en la comunicación terapéutica puesto se percibe violenta.

Finalmente, el hecho de que el personal continúe contemplando la sexualidad de las jóvenes como un asunto que compete a decisiones de los padres, se contrapone con lo que se ha proclamado sobre la autonomía de las adolescentes en materia de sexualidad y reproducción. Fueron recurrentes las narrativas que evidenciaron la violación continua y sistemática de sus derechos sexuales y reproductivos, lo que es particularmente grave en un país que apenas en 2013 suscribió el Consenso de Montevideo sobre Población y Desarrollo, instrumento internacional que reconoce (por primera vez) en América Latina (AL) y El Caribe los derechos sexuales como Derechos Humanos (CEPAL, 2013).

\section{Consideraciones finales}

Los resultados que se han expuesto derivan de un acercamiento riguroso desde lo metodológico, habiendo procurado evidencias de orígenes distintos que han posibilitado la triangulación y validación de los datos obtenidos. En el sentido anterior y sin embargo, los resultados no deberían generalizarse en función de que el estudio tuvo lugar en municipios de tipo urbano y jóvenes que continúan insertas en el sistema educativo (modalidad escolarizada y no escolarizada).

En esta investigación se documenta la existencia de una serie de limitaciones para la prevención del embarazo subsiguiente en madres adolescentes que reciben apoyo para continuar incorporadas al sistema educativo. Entre dichas limitaciones se encuentran aquellas que derivan directo de cómo se operativizan las políticas institucionales, de las competencias profesionales que posee el personal de salud para implementar la denominada "atención amigable" y de los imaginarios morales que posee también este personal. La convergencia de las limitantes antes señaladas deriva en que estas jóvenes, aunque en su mayoría se encuentran protegidas mediante el uso de un método anticonceptivo, hagan uso de este a partir de una experiencia enmarcada en el desconocimiento, insatisfacción y comodidad, lo que no contribuye a su empoderamiento sexual y reproductivo.

\section{Referencias}

ALEMAN, X.; INSFRÁN, S.; CASTILLO, A. Embarazo a edad temprana y en adolescentes. ¿Porqué y cómo debemos prevenirlo en América Latina? Washington, DC: Banco Interamericano del Desarrollo, 2018. Disponível em:

<https://bit.ly/2XOsi5W>. Acesso em: 24 out. 2018.

ANGROSINO, M. La recogida de datos en el campo. In: ANGROSINO, M. (Ed.). Etnografía y observación participante en investigación cualitativa. San Sebastián de los Reyes: Morata, 2012. p. 58-78.

BALDWIN, M. K.; EDELMAN, A. B. The effect of long-acting reversible contraception on rapid repeat pregnancy in adolescents: a review. Journal of Adolescent Health, São Francisco, v. 52, n. 4, p. 47-53, 2013. Disponível em: <https://bit.ly/3hfipWs>.

Acesso em: 3 mar. 2018.

BARROS, X. Educación, expectativas y salud: relación virtuosa dirigida a adolescentes en situación de vulnerabilidad. Revista Cuadernos de Inclusión en Educación Superior, Santiago, n. 3 ,

p. 22 35, 2019. Disponível em: <https://bit.ly/3dWUI3i>. Acesso em: 10 jun. 2020.

BENAVIDES, F.; DELCLÓS, J.; CONSOL, R. Estado del bienestar y salud pública, una relación que debe ser actualizada. Gaceta Sanitaria, Amsterdã, v. 32, n. 2, p. 193-197, 2018. Disponível em: <https://bit.ly/2YkSp3f>. Acesso em: 12 out. 2018.

BINSTOCK, G.; CERRUTTI, M. Carreras truncadas. El abandono en el nivel medio en Argentina.

Buenos Aires: Unicef, 2005.

CEPAL - COMISIÓN ECONÓMICA PARA AMÉRICA LATINA Y EL CARIBE. Primera reunión de la Conferencia Regional sobre Población y Desarrollo de América Latina y el Caribe. Montevidéu, 12-15 ago. 2013. Disponível em: <https://bit.ly/2XPK5JZ>. Acesso em: 10 jun. 2020.

CONAPO - CONSEJO NACIONAL DE POBLACIÓN. Aspectos a considerar en la 
construcción de indicadores sobre fecundidad y salud sexual y reproductiva a partir de la Encuesta Nacional de la Dinámica Demográfica, 2014. In: CONAPO - CONSEJO NACIONAL DE POBLACIÓN. Situación de la Salud Sexual y Reproductiva. Cidade do México, 2016. p. 19-44.

DOUGLAS, M. La aceptabilidad del riesgo según las ciencias sociales. Barcelona: Paidós, 1982.

ESCOBAR, J. Prevención del embarazo subsecuente, una tarea impostergable. In: CURSO INTERNACIONAL DE ADOLESCENCIA SAIA-HGOIA, 5., 2006, Quito. Anais... [S.l.: s.n.], 2006. Disponível em: <https://bit.ly/2YmufoX>. Acesso em: 18 out. 2018.

GIDDENS, A. The constitution of society. Cambridge: Polity Press, 1986.

GÓMEZ, R. et al. Acciones educativas dirigidas a mejorar la percepción de riesgo del embarazo en adolescentes. Revista Cubana de Salud Pública, Cuba, v. 43, n. 2, p. 180-19o, 2017. Disponível em: <https://bit.ly/3cSqgpv>. Acesso em: 13 set. 2018.

GONZÁLEZ, E. Embarazo repetido en la adolescencia: la realidad chilena. Revista chilena de obstetricia y ginecología. Santiago, v. 81, n. 5, p. 354-359, 2016. Disponível em: <https://bit.ly/2UtQvfJ>.

Acesso em: 13 set. 2018.

JIMÉNEZ, A.; GRANADOS, J. A.; ROSALES, R. A. Embarazo en adolescentes de una comunidad rural de alta marginalidad. Un estudio mixto de caso. Salud Pública de México, Cuernavaca, v. 59, p. 1118, 2017. Disponível em: <https://bit.ly/37jfcko>. Acesso em: 18 ago. 2018.

MEDINA, O. S.; ORTIZ, K. Fecundidad en adolescentes y desigualdades sociales en México. Revista Panamericana de Salud Publica, Washington, DC, v. 42, p. 1-8, 2018.

MENDOZA, T.; ARIAS, M.; MENDOZA, LI. Hijo de madre adolescente: riesgos, morbilidad y mortalidad neonatal. Revista chilena de obstetricia y ginecología, Santiago, v. 77, n. 5, p. 375-382, 2012. Disponível em: <https://bit.ly/3hinuo3>. Acesso em: 10 jun. 2020.

MÉXICO. Secretaría de Salud. Instituto Nacional de Salud Pública. Encuesta Nacional de Saludy
Nutrición 2012. Curnavaca: Centro de Investigación em Evaluación y Encuestas, 2012. Disponível em: <https://bit.ly/3f9OTiR>. Acesso em: 10 set. 2018.

MÉXICO. Gobierno de la República. Estregia Nacional para la Prevención del Embarazo en Adolescentes. Cidade do México, 2015. Disponível em: <https://bit.ly/2YhI5Jk>. Acesso em: 10 set. 2018.

NÚÑEZ, G.; AYALA, D. Embarazo adolescente en el noroeste de México: entre la tradición y la modernidad. Culturales, Baja California, v. 8, n. 15, p. 7-46, 2012. Disponível em: <https://bit.ly/3dTssyj>. Acesso em: 13 jul. 2018.

PACHECO, C. Pregnancy under fifteen: the motives and redefinition of life's course. Salud Pública de México, Cuernavaca, v. 58, n. 1, p. 56-61, 2016. Disponível em: <https://bit.ly/37hjgRQ>. Acesso em: 10 jun. 2020.

PEÑARANDA, C. Salud Pública, justicia social e investigación cualitativa: hacia una investigación por principios. Revista Facultad Nacional de Salud Pública, Medelín, v. 33, n. 1, p. 106-114, 2015.

RAIDOO, S. et al. Dual-method contraception among adolescents and young people: are longacting reversible contraception users different? A qualitative study. Journal of Pediatric and Adolescent Gynecology, Amsterdã, v. 33, n. 1, p. 45-52, 2020. Disponível em: <https://bit.ly/2zpOdXE〉.

Acesso em: 10 jun. 2020.

RODRÍGUEZ, J. La reproducción en la adolescencia en América Latina: viejas y nuevas vulnerabilidades. Revista Internacional de Estadística y Geografía, [S.l.], v. 3, n. 2, p. 66-81, 2012. Disponível em: <https://bit.ly/3cNfAbD>. Acesso em: 10 jun. 2020.

ROJAS RAMÍREZ, G. et al. Acceso a anticoncepción en adolescentes: percepciones de trabajadores de la salud en Huechuraba, Chile. Revista Panamericana de Salud Pública, Washington, DC, v. 41, p. 77, 2017. Disponível em: <https://bit.ly/3cUCFZT>. Acesso em: 19 set. 2018.

ROSAS, A. et al. Reembarazo en la adolescencia: análisis de la población perteneciente a la Red del Hospital Padre Hurtado, entre los años 2005-2015. Revista chilena de obstetricia y ginecología, 
Santiago, v. 82, n. 4, p. 361-372, 2017. Disponível em: <https://bit.ly/3dQnMcH>. Acesso em: 13 jul. 2018.

SAM, S. et al. Comportamiento sexual y anticoncepción en la adolescencia. Acta Pediátrica de México, Cidade do México, v. 35, p. 49o-498, 2014. Disponível em: <https://bit.ly/3dRwCH2>. Acesso em: 10 jul. 2018.

SILVA, N. et al. Análisis de partos en adolescentes y repercusiones perinatales en una maternidad pública en el Amazonas. Adolescência \& Saúde, Rio de Janeiro, v. 15, n. 1, p. 50-57, 2018. Disponível em: <https://bit.ly/2MJ99Ml>. Acesso em: 10 out. 2018.

SOLÍS, O.; SILVA, J. A. Discursos del cuerpo; anticonceptivos y control natal. México al comenzar la década de los setenta. CONAMED, Cidade do México, v. 22, n. 4, p. 197-198, 2017. Disponível em: <https://bit.ly/2Yo78dS>. Acesso em: 21 set. 2018.

STRAUSS, A., CORBIN, J. Bases de la investigación cualitativa, técnicas y procedimientos para desarrollar la teoría fundamentada. Bogotá, DC: Ed. Universidad de Antioquia, 2002.

TAQUETTE, S. R. Derechos sexuales y reproductivos en la adolescencia. Adolescência \& Saúde, Rio de Janeiro, v. 10, p. 72-77, 2013. Suplemento 1. Disponível em: <https://bit.ly/2YlzyoJ>. Acesso em: 10 out. 2018.
UNFPA - FONDO DE POBLACIÓN DE LAS

NACIONES UNIDAS. Maternidad y unión en niñas $y$ adolescentes: consecuencias en la vulneración de sus derechos. Informe final. São Salvador, 2016. Disponível em: <https://bit.ly/2Yk1Sbb>.

Acesso em: 10 jun. 2020.

VALENZUELA, C.; VILLAVICENCIO, L. La constitucionalización de los derechos sexuales y reproductivos: hacia una igual ciudadanía para las mujeres. Ius et Praxis, Talca, v. 21, n. 1, p. 271-314, 2015. Disponível em: <https://bit.ly/3owYTyG>. Acesso em: 7 set. 2018.

VILLALOBOS, A. et al. Embarazo adolescente y rezago educativo: análisis de una encuesta nacional en México. Salud Pública de México, Cuernavaca, v. 57, p. 135-143, 2015. Disponível em: <https://bit.ly/2zkTJdY>. Acesso em: 12 maio 2018.

WHO - WORLD HEALTH ORGANIZATION. Making health services adolescent friendly: developing national quality standards for adolescent-friendly health services. Genebra, 2012. Disponível em: <https://bit.ly/3dXh44I >. Acesso em: 10 jun. 2020.

ZAMBERLIN, N. et al. Adopción y adherencia en el uso de métodos anticonceptivos posevento obstétrico en adolescentes de 14-20 años. Revista Argentina de Salud Pública, Buenos Aires, v. 8, n. 32, p. 26-33, 2017. Disponível em: <https://bit.ly/2XUhOC5>. Acesso em: 25 ago. 2018.

\section{Agradecimientos}

A las jóvenes que con sus experiencias posibilitan comprender en mayor profundidad sus necesidades particulares, a la Secretaria Estatal de Educación Pública del Estado de San Luis Potosí por brindar las facilidades para contactar a las participantes.

\section{Contribución de los autores}

González Nava propuso la idea original y junto con Rangel Flores construyó el protocolo de investigación. Las entrevistas fueron realizadas por Nava y el análisis se llevó bajo la guía de Rangel Flores. Hernández Ibarra contribuyó en el diseño e implementación de la metodología cualitativa. Todos contribuyeron en la discusión teórica de los resultados y en la elaboración del artículo científico.

Recibido: 04/03/2020

Re-presentado: 31/03/2020

Aprobado: 22/04/2020 